\title{
A Stone-Weierstrass theorem for random functions
}

\section{A. Mukherjea}

\begin{abstract}
It is shown in this note that if $Q$ is an algebra of uniformly bounded mean-square continuous real-valued random functions indexed in a compact set $T$, containing all bounded random variables and separating points of $T$ (i.e., given $t_{1}$ and $t_{2}$ in $T$, there is a random function $X_{t}$ in $Q$ such that $\left|X_{t_{1}}-X_{t_{2}}\right|=1$ ), then given any mean square continuous random function, there is a sequence in $Q$ converging in mean square to the given random function uniformly on $T$.
\end{abstract}

The purpose of this note is to present a Stone-Weierstrass type theorem for random functions which might find possible future applications in probability theory or analysis. Tzannes in [2] showed that a mean square continuous (m.s.c.) second order random function (r.f.) can be approximated uniformly in mean square by a sequence of random polynomials (i.e., polynomials with random variables as co-efficients). So it is natural to consider the same problem in the more general situation which we describe in the following paragraph.

Let $T$ be a compact set in some topological space. Let us restrict our attention to real-valued random functions on some probability space indexed in the parameter set $T$. A r.f. $X_{t}$ is said to be m.s.c. on $T$ if for every $t$ in $T, E\left[X_{t}^{2}\right]=\int X_{t}^{2}<\infty$ and

Received 22 December 1969. 
and $n\left(x_{t}-X_{s}\right)=\left\{\int\left[X_{t}-X_{s}\right]^{2}\right\}^{\frac{1}{2}}$ tends to 0 as $s$ tends to $t$. A r.f. $X_{t}$ is uniformly bounded if there is a constant $M$ such that for every $t,\left|x_{t}\right|<M$. A family $Q$ of random functions is called an algebra if

(i) $X_{t}$ and $Y_{t}$ in $Q$ implies that $X_{t} \cdot y_{t}$ (pointwise multiplication) is also in $Q$ and

(ii) $X_{t}$ and $Y_{t}$ in $Q$ implies that $X_{t}+y_{t}$ is also in $Q$.

The uniformly bounded m.s.c. random functions can be easily seen to form an algebra. $Q$ is said to separate points in $T$ if given $t_{1}$ and $t_{2}$ in $T$, there exists a r.f. $X_{t}$ in $Q$. such that $\left|X_{t_{1}}-X_{t_{2}}\right|=1$. If $T=[0,1]$, the algebra of random polynomials separate points of $T$. This is the desired Stone-Weierstrass setting in which we consider the problem mentioned in the first paragraph. We have, as can be expected, the following theorem.

THEOREM. Let $Q$ be an algebra of uniformly bounded m.s.c. random functions containing all bounded random variables. Let $Q$ also separate points of $T$. Then given a m.s.c. r.f., there exists a sequence in $Q$ which converges in mean square to the given r.f. uniformly on $T$.

Proof. The proof follows closely the classical pattern.

Following the classical proof (see page 131, [1]), one can easily check that if $X_{t}$ is in $Q$, then $\left|X_{t}\right|$ is in $\bar{Q}$, the closure of $Q$ in the uniform mean square limit sense.

Next, given $t_{1}$ and $t_{2}$ and any two random variables $X_{1}$ and $X_{2}$ in $Q$, we can find $z_{t}$ in $\bar{Q}$ such that $z_{t_{1}}=X_{1}$ and $z_{t_{2}}=X_{2}$; for we can take $2_{t}=X_{1}+\left|X_{t}-X_{t_{1}}\right| \cdot\left(X_{2}-X_{1}\right)$ where $X_{t}$ is in $Q$ such that $\left|x_{t_{1}}-x_{t_{2}}\right|=1$.

Now let $W_{t}$ be any m.s.c. non-negative r.f. . We wish to show that $W_{t}$ is in $\bar{Q}$. With no loss of generality, we can assume that $W_{t}$ is uniformly bounded. For, given $\varepsilon>0$, using the mean square continuity 
of $W_{t}$ and the r.f. $W_{t m}=\inf \left\{m, W_{t}\right\}$, where $m$ is a constant, and the compactness of $T$, we can find a $m$ such that $n\left(W_{t}-W_{t m}\right)<\varepsilon$ for every $t$ in $T$. [Note that here $n$ denotes the $L_{2}$-norm.]

So we assume that $W_{t}$ is uniformly bounded by a constant $m$. Let $I_{D}$ be the characteristic function of the measurable set $D$ and so it is a random variable in $Q$. Let $t_{0}$ be in $T$. Then for every $t^{\prime}$ in $T$, we can find a neighbourhood $N_{t^{\prime}}$ of $t^{\prime}$ and $y_{t}^{t^{\prime}}$ in $\bar{Q}$ such that $y_{t_{0}}^{t^{\prime}}=W_{t_{0}}$ and $n\left(Y_{t}^{t^{\prime}} \cdot I_{D}\right)<n\left(W_{t} \cdot I_{D}\right)+\varepsilon / 3 m$ for every $t$ in $N_{t^{\prime}}$ and every measurable set $D$. Then using the compactness of $T$ and noting that $\inf \left\{X_{t}, y_{t}\right\}$ is in $\bar{Q}$ for $X_{t}$ and $y_{t}$ in $\bar{Q}$, we can find a $y_{t}^{t_{0}}$ in $\bar{Q}$ such that

$$
y_{t_{0}}^{t_{0}}=W_{t_{0}} \text { and } n\left(Y_{t}^{t_{0}^{0}} \cdot I_{D}\right)<n\left(W_{t} \cdot I_{D}\right)+\varepsilon / 3 m
$$

for every $t$ in $T$ and every measurable set $D$. Now we can find a neighbourhood $N_{t_{0}}$ of $t_{0}$ such that for every $t$ in $N_{t_{0}}$ and every measurable set $D$,

$$
n\left(Y_{t}^{t_{0}} \cdot I_{D}\right)>n\left(W_{t} \cdot I_{D}\right)-\varepsilon / 3 m \text {. }
$$

Doing this for every $t_{0}$ in $T$, then we can find a $y_{t}$ in $\bar{Q}$ such that $\left|n\left(Y_{t} \cdot I_{D}\right)-n\left(W_{t} \cdot I_{D}\right)\right|<\varepsilon / 3 m$ for every $t$ in $T$ and every measurable set $D$. Then $\left|E\left(Y_{t}^{2} \cdot I_{D}\right)-E\left(W_{t}^{2} \cdot I_{D}\right)\right|<\varepsilon$. Now let $A_{t}=\left[W_{t} \geqq Y_{t}\right]$. Then

$$
n\left(Y_{t}-W_{t}\right) \leqq n\left(I_{A_{t}} \cdot\left(Y_{t}-W_{t}\right)\right)+n\left(I_{A} c \cdot\left(Y_{t}-W_{t}\right)\right),
$$

each of which is less than $\sqrt{\varepsilon}$; for 


$$
\begin{aligned}
& E\left(I_{A_{t}} \cdot\left(Y_{t}-W_{t}\right)^{2}\right)=E\left(W_{t}^{2} \cdot I_{A_{t}}\right)+E\left(Y_{t}^{2} \cdot I_{A_{t}}\right)-2 E\left(Y_{t} \cdot W_{t} \cdot I_{A_{t}}\right) \\
& \leqq E\left(W_{t}^{2} \cdot I_{A_{t}}\right)-E\left(Y_{t}^{2} \cdot I_{A_{t}}\right)<\varepsilon
\end{aligned}
$$

and similarly the other one.

Finally, let $W_{t}$ be any m.s.c. r.f. . Then since $T$ is compact and $W_{t}$ is m.s.c. given $\varepsilon>0$, we can find $\beta>0$ such that $P(B)<\beta$ (where $P$ is the measure in the probability space) implies that $n\left(W_{t} \cdot T_{B}\right)<\varepsilon$ for every $t$ in $T$. Noting that $E\left(W_{t}^{2}\right)$ is a bounded function of $t$, we can find a number $k>0$ such that for every $t$ in $T$, there is a $B_{t}$, a measurable set such that $P\left(B_{t}\right)<\beta$ and on $B_{t}^{c}$, $\left|W_{t}\right|$ is less than $k$. Then we write $U_{t}=\sup \left\{-k, \inf \left(W_{t}, k\right)\right\}$ so that $U_{t}$ is clearly a m.s.c. r.f. bounded by $k$ for all $t$. We note that on $B_{t}^{c}, U_{t}=W_{t}$ and therefore, since $\left|U_{t}\right| \leqq\left|W_{t}\right|$, it is easy to see that $n\left(W_{t}-U_{t}\right)=n\left(I_{B_{t}} \cdot\left(W_{t}-U_{t}\right)\right)<2 \varepsilon$. Now $k-U_{t}$ is a non-negative m.s.c. r.f. and so we can find $y_{t}$ in $\bar{Q}$ such that $n\left(U_{t}-\left(k-Y_{t}\right)\right)<\varepsilon$ and this proves that there is a $z_{t}=k-y_{t}$ in $\bar{Q}$ such that $n\left(W_{t}-Z_{t}\right)<3 \varepsilon$ for every $t$ in $T$. This completes the proof of the theorem.

\section{References}

[1] J. Dieudonné, Foundations of modern analysis (Academic Press, New York, London, 1960).

[2] Nicolaos S. Tzannes, "Polynomial expansions of random functions", IEEE Trans. Information Theory IT-13 (1967), 314.

University of South Florida, Tampa, Florida, USA. 